\title{
Assessment of cutaneous sensory and autonomic axon reflexes in rheumatoid arthritis
}

\author{
V A Jolliffe, P Anand, B L Kidd
}

\begin{abstract}
Objective-To assess sensory function in skin overlying the joints of patients with rheumatoid arthritis, in relation to the pain and tenderness which commonly arises in structures not directly involved in the inflammatory process.

Methods-An intradermal injection of capsaicin $0.05 \mu \mathrm{g}$ in $10 \mu \mathrm{l}$ was made over the wrists and forearms of 40 patients with rheumatoid arthritis and 46 control subjects. Axon reflex vasodilatation was measured using laser Doppler flowmetry. Cholinergic sympathetic function was assessed by measuring axon reflex sweating induced by a single intradermal injection of nicotine $0.5 \mu \mathrm{g}$ in $0.1 \mathrm{ml}$.

Results-Capsaicin induced axon reflex vasodilatation over the wrists was found to decrease with age in normal subjects $(r=-0.62, p<0.001)$. In patients with rheumatoid arthritis, capsaicin induced axon reflex vasodilatation was significantly greater over the wrists, but not the forearms, when compared with age matched normal controls $(p<0 \cdot 01)$. A minimal correlation between axon reflex vasodilatation and visual analogue pain score was apparent in the rheumatoid group $(r=-0.37, p<0.05)$. Nicotine induced sweating responses were similar in the rheumatoid and normal groups, and both showed a linear age related decline.

Conclusions-The results show a selective increase of capsaicin induced vasodilatation in skin overlying joints in patients with rheumatoid arthritis. This suggests that the activity of a subpopulation of periarticular small sensory fibres is altered, which may explain, at least in part, some of the clinical findings in this disorder.
\end{abstract}

(Ann Rheum Dis 1995; 54: 251-255)

Inflammation

Research Group,

London Hospital

Medical College,

London E1 2AD,

United Kingdom

V A Jolliffe

B L Kidd

Neurosciences

Research Group $P$ Anand

Correspondence to: Dr B Kidd,

ARC Building,

London Hospital Medical College,

London E1 2AD

United Kingdom.

Accepted for publication 28 October 1994

Recent experimental studies have provided new insights into peripheral mechanisms involved in the generation of arthritic pain. ${ }^{1}$ Within the joint itself, inflammatory mediators fibres (nociceptors) which then respond to various chemical and mechanical stimuli to signal pain..$^{2-4}$ In contrast, the origin of the pain and tenderness (hyperalgesia) which commonly arises from periarticular structures not directly involved in the articular disease process remains unclear. Both peripheral and central mechanisms have been proposed, ${ }^{1}$ although direct evidence for either mechanism is lacking. The aim of this study was to seek evidence for altered peripheral function of small sensory fibres in periarticular tissues of patients with chronic rheumatic disease.

Detailed assessments of small sensory fibre function in rheumatoid arthritis have not been performed despite evidence of sensory neuropathy in many patients with this disorder. ${ }^{5}$ Capsaicin, the algesic substance in red peppers, has a selective action on small sensory fibres to produce pain and axon reflex vasodilatation $^{6}$ and has been used as a tool to examine sensory function in normal and pathological states. ${ }^{7-9}$ In degenerative disease of the spine, skin flares appear to be reduced, ${ }^{10}$ whilst in chronic musculoskeletal pain syndromes of non-inflammatory origin a generalised increase has been reported. ${ }^{11}$

In the present study we have examined small sensory and autonomic fibre function in the skin of patients with rheumatoid arthritis. Intradermal capsaicin was used to compare skin flares and pain over involved rheumatoid joints with control sites and with flares in normal individuals. Cholinergic sympathetic function was also assessed in the same regions by measuring nicotine induced axon reflex sweating, ${ }^{12}$ to exclude the presence of a generalised small fibre neuropathy.

\section{Methods}

SUBJECTS

A total of 86 subjects were involved in the study. Forty patients with rheumatoid arthritis as defined by the American Rheumatism Association criteria ${ }^{13}$ were chosen from those attending a rheumatology outpatient department. Control data were obtained from 46 subjects who were selected from hospital staff and relatives of patients. Approval for the study was granted by the relevant ethics committee and informed consent obtained before any procedure.

A history was taken from all subjects and appear to sensitise previously inactive sensory included details of past medical illness and current medication. Subjects taking adrenergic receptor blocking drugs and other vasoactive drugs were excluded, as were subjects with overt diabetes. Inspection was made of the skin overlying the wrists and subjects with eczema, psoriasis or surgical scars in this area were also excluded. Wrists and elbows were examined for tenderness and graded on a scale $0=$ no tenderness, $1=$ mild tenderness, $2=$ moderate or severe tenderness accompanied by facial grimacing or withdrawal of the limb. The rheumatoid group had blood taken for 
erythrocyte sedimentation rate (ESR) as a general marker of inflammation.

SENSORY STUDIES

Subjects were rested for 30 minutes in a quiet room maintained at a constant $22^{\circ} \mathrm{C}$; they had taken no recent food or drink. They were seated with the arm to be tested resting comfortably on a table, supported by a pillow. Sensation to light touch and pinprick at the sites to be tested were measured using cotton wool and a sterile pin, respectively. Cutaneous blood flow was measured using laser Doppler flowmetry. ${ }^{14} 15$ The study utilised a Periflux PF3 (LDPF3, Perimed) laser Doppler with a $632.8 \mathrm{~nm}$ helium neon laser and a PF314 angle probe (small). The laser light was transmitted to the skin via a probe consisting of three silica fibres (fibre separation of $0.25 \mathrm{~mm}$, core diameter $0.125 \mathrm{~mm}$ ) with a sampling area over the skin surface of approximately $1 \mathrm{~mm}$ diameter and $1 \mathrm{~mm}$ depth. The reflected light was collected by a sensitive photoelectric detector with the voltage signal in $\mathrm{mV}$ (expressed as units of 'flux') being proportional to the velocity and number of the red blood cells within the intravascular space. ${ }^{15}$ Before each study the instrument was calibrated by setting output to zero while the probe was directed away from any reflecting surface.

The Doppler probe was placed on the skin, supported by a semicircular disc and secured with a double sided adhesive ring. This enabled accurate positioning of capsaicin injection adjacent to the Doppler probe without disturbing the position of the probe. Two sites were assessed, including the dorsum of the wrist and the mid volar forearm at a point equidistant from the olecranon and ulnar styloid.

A solution of capsaicin (Sigma) $5 \mu \mathrm{g} / \mathrm{ml}$ was made using Tween 80 and normal saline according to a standard method, ${ }^{16}$ and was filtered through a $0.02 \mu \mathrm{m}$ Anatop microfilter. Before the injection, baseline cutaneous laser Doppler measurements were recorded for one minute. Capsaicin $0.05 \mu \mathrm{g}$ in $10 \mu \mathrm{l}$ was then injected intradermally using a U-100 $0.5 \mathrm{ml}$ syringe (Terumo), ensuring that a good 'bleb' was produced by each injection. The dose chosen corresponded to the midpoint of a previously obtained dose response curve and allowed for increased or decreased responses to be observed. The injection was situated $2 \mathrm{~mm}$ medial to the tip of the probe. Results obtained from subjects in whom no bleb was observed were discarded. The pen recorder was kept running for the duration of the test at $1 \mathrm{~cm} / \mathrm{min}$.

Before starting the study, subjects were instructed in the use of a visual analogue scale (VAS) and were requested to score the most intense pain felt from the injection of capsaicin. A purpose built instrument was used, comprising a freely moving pointer with $100 \mathrm{~mm}$ of travel, marked at one end by the term 'no pain' and at the other by 'worst pain ever'. Gradations of $100 \mathrm{~mm}$ (not seen by the subject) were marked on the underside of the slide. The VAS was placed within easy reach of the upper limb not being assessed. The reproducibility of the techniques was assessed in 10 normal volunteers by comparing results of repeat testing one week apart.

\section{SWEATING STUDIES}

Nicotine for injection was diluted with sterile saline immediately before use, from a stock solution of nicotine acid tartrate prepared by University College Hospital Pharmacy.

Conditions for this part of the study were as for the sensory tests. An intradermal injection of $0.1 \mathrm{ml}(0.5 \mu \mathrm{g})$ of nicotine acid tartrate was administered using a U-100 $0.5 \mathrm{ml}$ syringe (Terumo). Optimum nicotine dosage was chosen after dose responses were performed on a small group of control subjects.

This test was performed on 25 rheumatoid patients and 25 control subjects over the dorsum of the wrist. Before testing, the area was cleaned using an alcohol wipe, to remove any surface grease that may have influenced the recordings of sweat rate, and the area was allowed to dry thoroughly. Peak rate of sweating was assessed using an evaporimeter resting on the skin surface over the injection site. ${ }^{12}$ Baseline measurements were taken after the evaporimeter had been in situ for at least 30 seconds before the injection of nicotine. An intradermal injection was then performed and the probe resited immediately adjacent to the site of injection. Recordings were taken every 15 seconds for the first minute and subsequently every 30 seconds until the sweat rate returned to within $10 \%$ of baseline.

\section{STATISTICS}

Data assessment was performed using a statistical software package (Statsgraphics: version 5). All results are expressed as mean (1 SE). Comparison of two groups was performed using a Student's $t$ test, after data were checked for normal distribution, with $\mathrm{p}<0.05$ considered significant. ANOVA testing was used for comparison of more than two variables.

\section{Results}

SENSORY STUDIES

Capsaicin induced vasodilatation in 10 normal volunteers tested on two occasions one week apart showed high reproducibility $(r=-0.72$, $\mathrm{p}<0.001$ ).

In normal subjects there was an age related decrease in axon reflex vasodilatation over the wrist joint $(r=-0.62, \mathrm{p}<0.001)$ (fig 1). In contrast, no correlation was found between age and axon reflex vasodilatation in the rheumatoid group $(r=+0.06, \mathrm{p}=0.73)$. There was significantly increased axon reflex vasodilatation over the wrists of patients with rheumatoid arthritis compared with normal control subjects $(t=-3 \cdot 32, \mathrm{p}=<0.01)$ (fig 2$)$.

There was no correlation between axon reflex vasodilatation and clinical signs of joint disease. Specifically, wrist tenderness in the rheumatoid group as assessed by the two point 


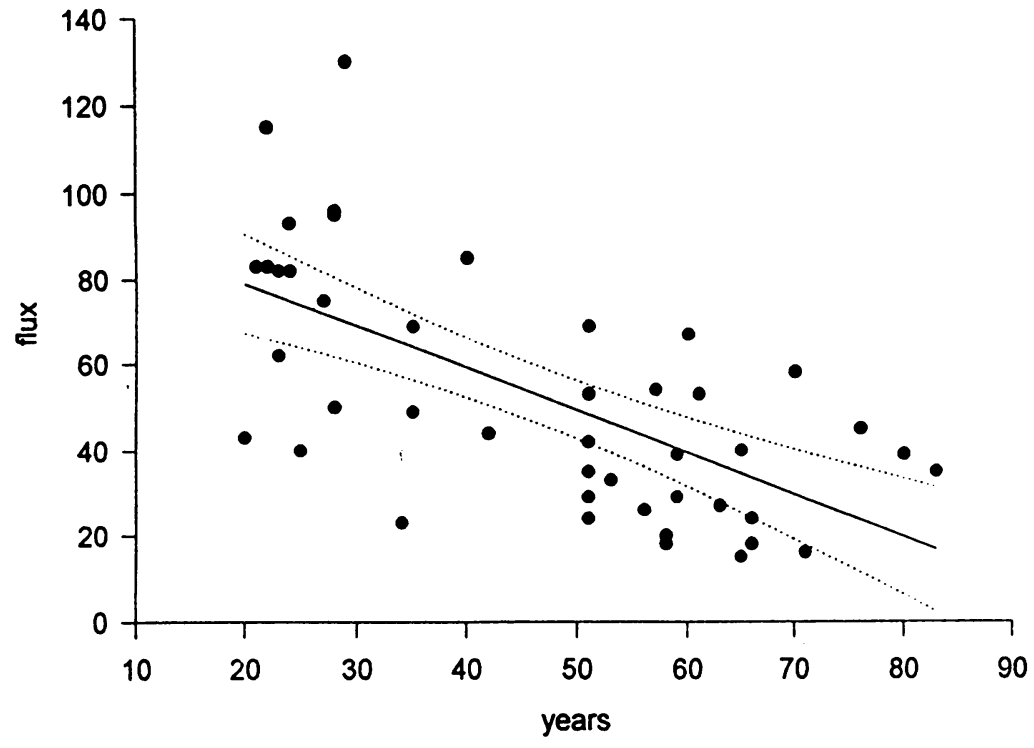

Figure 1 Capsaicin induced vasodilatation (expressed as arbitrary units of flux) compared with age in years in 46 control subjects. Solid line represents mean linear regression $(\mathrm{r}=-0.72 ; p<0.001)$; dotted line represents $99 \%$ confidence intervals of the mean.

scale did not correlate with capsaicin induced changes in blood flux recordings (table). Similarly, there was no correlation between axon reflex vasodilatation and ESR $(r=0.07$, $\mathrm{p}=0.68)$.

Cutaneous axon reflex vasodilatation was compared in the wrists and forearms of a subset of 25 subjects with rheumatoid arthritis and 25 age matched (but not sex matched)

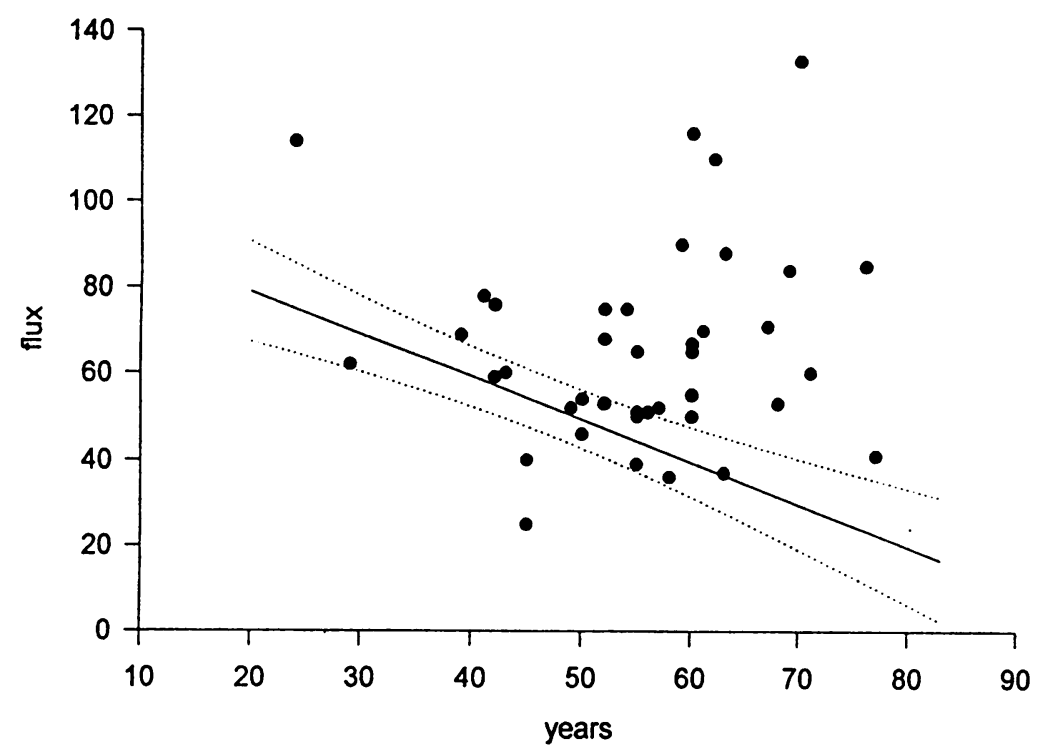

Figure 2 Capsaicin induced vasodilatation compared with age in years in 40 subjects with rheumatoid arthritis. Solid and dotted lines represent mean linear regression with $99 \%$ confidence intervals for data obtained from 46 normal subjects tested using an identical protocol (see fig 1).

Comparison of clinical parameters with capsaicin induced vasodilatation and pain in patients with rheumatoid arthritis

\begin{tabular}{lllll}
\hline Grade† & Number & $\begin{array}{l}\text { ESR } \\
(\mathrm{mm} / 1 \mathrm{st} h)\end{array}$ & $\begin{array}{l}\text { Capsaicin induced } \\
\text { vasodilatation } \\
\text { (flux) } \neq\end{array}$ & $\begin{array}{l}\text { Capsaicin induced } \\
\text { VAS pain score }\end{array}$ \\
\hline 0 & 15 & $36 \cdot 4(10 \cdot 3)$ & $63 \cdot 6(6 \cdot 5)$ & $52 \cdot 3(6 \cdot 1)$ \\
1 & 12 & $40 \cdot 5(10 \cdot 9)$ & $61 \cdot 6(6 \cdot 5)$ & $61 \cdot 9(7 \cdot 8)$ \\
2 & 13 & $57 \cdot 3(12 \cdot 2)$ & $62 \cdot 3(6 \cdot 7)$ & $51 \cdot 8(7 \cdot 5)$ \\
\hline
\end{tabular}

Values are mean (SE). +Grade $0=$ no joint tenderness, $1=$ mild tenderness, $2=$ moderate or severe tenderness. $¥$ Arbitrary units. control subjects. The mean age was $57 \cdot 2(2 \cdot 1)$ years in the rheumatoid group and $57 \cdot 4(2 \cdot 1)$ years in the control group. Baseline blood flux recordings were alike over the forearms and wrists of the rheumatoid and control subjects. Capsaicin induced axon reflex vasodilatation was similar in the forearms of the rheumatoid and control subjects, but was substantially greater in wrists of rheumatoid subjects (71.4 (5.2) units) compared with controls $(37 \cdot 6(3 \cdot 3)$ units) $(\mathrm{p}<0 \cdot 01)$ (fig 3$)$.

The mean VAS pain score was similar in both the rheumatoid subjects (55 (3)) and controls (54 (4)) $(\mathrm{p}=0.89)$. There was no correlation between VAS pain score and axon reflex vasodilatation in the control group $(r=+0.24, \mathrm{p}=0.1)$; however, a weak correlation was apparent in the rheumatoid group $(r=-0.37, \mathrm{p}<0.05)$.

All subjects in the rheumatoid group were taking non-steroidal anti-inflammatory drugs. In order to determine if these agents might influence the response to capsaicin, a group of six normal volunteers were given ibuprofen $400 \mathrm{mg}$ eight hourly for 24 hours and capsaicin responses were compared before and after the therapy period. Mean baseline blood flux recordings were similar before $(5.5(0.7)$ units $)$ and after $(6 \cdot 0(0 \cdot 6)$ units $)$ drug administration $(p=0.91)$. The magnitude of capsaicin induced vasodilatation was also similar before $(61.2(10 \cdot 5)$ units) and after (59.3 (10.1) units) drug administration $(p=0 \cdot 9)$. Ibuprofen did not significantly alter the VAS pain scores recorded by the subjects (64 (9) $v 58$ (7); $\mathrm{p}=0.66)$.

\section{SWEATING STUDIES}

An age related decrease in nicotine induced sweating was found in both the normal group $(r=-0.59, \mathrm{p}<0.01)$ and the rheumatoid group $(r=-0.58, \mathrm{p}<0.01)$. No differences were apparent between the normal or rheumatoid subjects $(t=-0 \cdot 70, \mathrm{p}=0.48)$ or between males and females $(t=-0.40, \mathrm{p}=0.68)$. In the rheumatoid group there was no correlation between sweating and axon reflex vasodilatation $(r=0.11, \mathrm{p}=0.67)$.

\section{Discussion}

The present study has shown increased vasodilatation in response to intradermal capsaicin over the joints of patients with rheumatoid arthritis. The increase was localised to the area overlying the joint and was not apparent at sites away from the joint, implying a regional alteration of capsaicin sensitive small fibre function in patients with inflammatory joint disease. This parallels observations made in experimental arthritis, where the majority of articular small afferent fibres have been shown to develop a long lasting 'sensitisation' after the onset of joint inflammation. ${ }^{2-4}$

Capsaicin is a naturally occurring substance that has direct excitatory actions on polymodal (or 'mechano-heat') classes of somatic and visceral small diameter sensory nerve fibres. These fibres serve to transmit nociceptive 


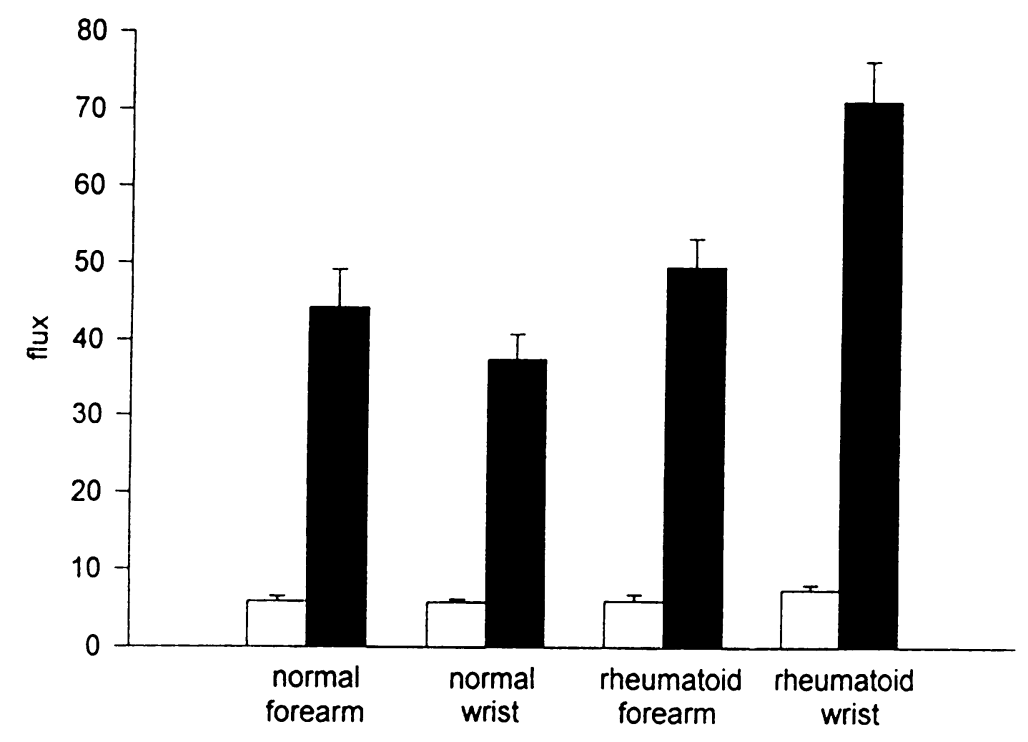

Figure 3 Mean (SEM) unstimulated ( $\square$ ) and capsaicin induced ( $\mathbf{0}$ ) blood flow (expressed as arbitrary units of flux) in the forearms and wrists of 24 age matched normal subjects and 24 patients with rheumatoid arthritis. Significant difference $(p<0.01)$ between stimulated blood flow over the wrists of rheumatoid patients and that in normal subjects.
In agreement with previous studies, ${ }^{12}$ we found that nicotine induced axon reflex sweating decreased with age. Disturbances of autonomic activity have been observed in rheumatoid arthritis ${ }^{19} 20$ which may be associated with clinical sensory neuropathy. ${ }^{19}$ In the present study, however, there was no clinical evidence of peripheral neuropathy among the rheumatoid patients. Decreased sweat area in females with rheumatoid arthritis has been reported, ${ }^{21}$ but we were unable to confirm this. Alterations of sympathetic vasomotor activity have been correlated with persistence of pain in the musculoskeletal disorder frozen shoulder, ${ }^{22}$ but no alteration of sympathetic (cholinergic) activity was observed in the present study.

The mechanism involved in the increased capsaicin induced vasodilatation in skin overlying joints in rheumatoid arthritis remains unclear. It is unlikely that this results from a generalised neuropathy or vasculopathy, as baseline and axon reflex vasodilatation was unaffected in skin not overlying joints. It is also unlikely that the increased vasodilatation was the result of generalised sympathetic dysfunction, ${ }^{23}{ }^{24}$ as we did not observe any change in axon reflex sweating. Although all the patients with rheumatoid arthritis were taking non-steroidal anti-inflammatory drugs, these agents, at least in the normal volunteers tested with ibuprofen, did not appear to influence the magnitude of the capsaicin induced response. Finally, it is possible that the laser beam may have penetrated through the skin and detected changes in capsular blood flow, although we believe this to be unlikely in view of the relatively short penetration of laser beams at the wavelength used.

Skin flares are mediated, at least partly, by the neuropeptides substance $\mathrm{P}$ and calcitonin gene related peptide (CGRP), ${ }^{25}$ and it is relevant that substance $P$ has been reported to be altered in skin tissue in an animal model of tissue inflammation. ${ }^{26}$ In turn, the expression of substance $P$ and CGRP appears to be regulated by nerve growth factor (NGF), ${ }^{27} 28$ as does capsaicin sensitivity of sensory neurones. ${ }^{29}$ There is a report of increased concentrations of NGF in the synovial fluid of patients with rheumatoid arthritis ${ }^{30}$ and a similar increase in skin overlying inflamed joints would explain the findings of the present study. Such an increase might arise as a result of a purely peripheral mechanism following regional increases in mediators released from inflamed joints. There was, however, no correlation between ESR or wrist joint tenderness and capsaicin induced vasodilatation, as might have been expected if this were the case.

A second possibility is that the observed changes were secondary to a central spinal mechanism. Convergence of wrist joint afferents and wrist cutaneous afferents potentially might alter NGF expression in the latter, thereby producing sensitisation. Such synaptic connections could outlast the acute inflammatory response, leading to prolonged effects. Such a mechanism remains highly speculative, how ever, and awaits the results of further studies. groups reported similar mean pain magnitude scores in response to the standard dose of capsaicin. These results suggest a dissociation between sensation and axon reflex vaso dilatation in chronic inflammatory disease. 
1 Schaible H G, Grubb B D. Afferent and spinal mechanisms of joint pain. Pain 1993; 55: 5-54.

2 Coggeshall R E, Hong K A P, Langford L A, Schaible H-G Schmitt R F. Discharge characteristics of fine medial articular afferents at rest and during passive movements of inflamed knee joints. Brain Res 1983; 272: 185-8.

3 Schaible H G, Schmidt R F. Effects of an experimental arthritis on the sensory properties of fine articular afferent units. $\mathcal{F}$ Neurophysiol 1985; 54: 1109-22.

4 Schaible H G, Schmidt R F. Time course of mechanosensitivity changes in articular afferents during developing experimental arthritis. $\mathcal{f}$ Neurophysiol 1988 60: $2180-95$.

5 Hart F D, Golding J R, Mackenzie D H. Neuropathy in rheumatoid disease. Ann Rheum Dis 1957; 16: 471-80.

6 Holzer P. Capsaicin: Cellular targets, mechanisms of action and selectivity for thin sensory neurons. Pharmacol Rev 1991; 43: 143-201.

7 Jancso N, Jancso-Gabor A, Szolcsanyi J. Direct evidence for neurogenic inflammation and its prevention by
denervation and by pretreatment with Capsaicin. $B r f$ Pharm Chemother 1967; 31: 138-51.

8 Jancso G, Janka Z. A simple test for topographical diagnosis of central nervous system lesions. Eur Neurol 1981; 20: 84-7.

9 Helme R D, McKernan S. Neurogenic flare responses following topical application of capsaicin in humans. Ann Neurol 1985; 18: 505-9.

10 LeVasseur S A, Gibson S J, Helme R D. The measurement of capsaicin-sensitive sensory nerve fibre function in elderly patients with pain. Pain 1990; 41: 19-25.

11 Helme R D, Littlejohn G O, Weinstein C. Neurogenic flare responses in chronic rheumatic pain syndromes. Clin Exp Neurol 1987; 23: $91-4$.

12 Collins K J, Weiner J S. Axon reflex sweating. Clin Sci 1961; 21: 333-44.

13 Arnett F C, Edworthy S M, Bloch D A, et al. The American Rheumatism Association 1987 revised criteria for the classification of Rheumatoid Arthritis. Arthritis Rheum 1988; 31: 315-24

14 Low P A, Caskey P E, Tuck R R, Fealey R D, Dyck P J Quantitative sudomotor axon reflex test in normal and neuropathic subjects. Ann Neurol 1983; 14: 573-80.

15 Parkhouse N, Le Quesne P M. Quantitative objective assessment of peripheral nociceptor C fibre function. f Neurol Neurosurg Psychiatry 1988; 51: 28-34.

16 Simone D A, Baumann T K, LaMotte $R$ H. Dosedependent pain and mechanical hyperalgesia in humans after intradermal injection of capsaicin. Pain 1989; 38: 99-107.

17 Lynn B. Capsaicin: actions on nociceptive C-fibres and therapeutic potential. Pain 1990; 41: 61-9.

18 Cervero F, Gilbert R, Hammond R G, Tanner J. Development of secondary hyperalgesia following nonpainful thermal stimulation of the skin: a psychophysical study in man. Pain 1993; 54: 181-9.

19 study in man. Pain 1993; 54: 181-9. rheumatoid arthritis. Ann Rheum Dis 1965; 24: 161-8.

20 Leden I, Eriksson A, Lilja B, Sturfelt G, Sundkvist G. eden I, Eriksson A, Lilja B, Sturfelt G, Sundkvist G.
Autonomic nerve function in rheumatoid arthritis of varying severity. Scand $\mathcal{F}$ Rheum 1983; 12: 166-70.

21 Kalliomaki J L, Saarimaa H A, Toivanen P. Axon reflex sweating in rheumatoid arthritis. Ann Rheum Dis 1963; 22: $46-9$.

22 Mani R, Cooper C, Kidd B L, Cole J D, Cawley M I D The use of laser Doppler flowmetry and transcutaneous oxygen tension electrodes to assess local autonomic dysfunction in patients with frozen shoulder. $\mathcal{f} R$ Soc Med 1989; 82: $536-8$

23 Hornyak M E, Naver H K, Rydenhag B, Wallin B G Sympathetic activity influences the vascular axon reflex in Sympathetic activity influences the vascular axon
the skin. Acta Physiol Scand 1990; 139: 78-84.

24 Wallin B. Neural control of human blood flow. $\mathcal{f}$ Auton Nerv Syst 1990; 30 (suppl): 185-90.

25 Holzer P. Local effector functions of capsaicin-sensitive sensory nerve endings: involvement of tachykinins, calcitonin gene-related peptide and other neuropeptides. Neuroscience 1988; 24: 739-68.

26 Donnerer J, Schuligoi R, Stein C. Increased content and transport of substance $P$ and calcitonin gene-related peptide in sensory nerves innervating inflamed tissue: evidence for regulatory function of nerve growth factor in vivo. Neuroscience 1992; 49: 693-8.

27 Lindsay R M, Harmar A J. Nerve growth factor regulation expression of neuropeptide genes in adult sensory expression of neuropeptide gene
neurones. Nature $1989 ; 337: 362-4$.

28 Anand P, Rudge P, Mathias C J, et al. New autonomic and sensory neuropathy with loss of sympathetic adrenergic function and sensory neuropeptides. Lancet 1991; 337 1253-4.

29 Winter J, Forbes C A, Sternberg J, Lindsay R M. Nerve growth factor (NGF) regulates adult rat cultured dorsal root ganglion neurone responses to the excitotoxin capsaicin. Neuron 1988; 1: 937-81.

30 Aloe L, Tuveri M A, Carcassi U, Levi-Montalcini R. Nerve growth factor in the synovial fluid of patients with chronic arthritis. Arthritis Rheum 1992; 35: 351-5. 